\title{
Oral Squamous Cell Carcinoma with an Unusual Clinical Manifestation: A Case Report
}

\author{
Farnaz Falaki $^{1 *} \cdot$ Zahra Delavarian $^{2} \cdot$ Nooshin Mohtasham $^{3}$ \\ ${ }^{1}$ Assistant Professor, Department of Oral Medicine, Faculty of Dentistry and Dental Research Center, Mashhad University of Medical \\ Sciences, Iran \\ ${ }^{2}$ Associate Professor, Department of Oral Medicine, Faculty of Dentistry and Dental Research Center, Mashhad University of Medical \\ Sciences, Iran \\ ${ }^{3}$ Assistant Professor, Department of Oral and Maxillofacial Pathology, Faculty of Dentistry and Dental Research Center, Mashhad \\ University of Medical Sciences, Iran \\ *Corresponding Author; E-mail: falakif@mums.ac.ir
}

\begin{abstract}
Squamous cell carcinoma (SCC) is the most common malignant tumor of the oral cavity and one of the ten most common causes of death. It arises from dysplastic oral squamous epithelium. Considering the pathogenesis of SCC, a smooth and intact surface in this lesion is not a usual finding.

In this paper, we report an extremely rare case of oral SCC in buccal mucosa presenting as an exophytic lesion with smooth and intact surface, very unusual for oral SCC.
\end{abstract}

Key words: Oral SCC, oral cancer, oral malignancy, unusual clinical presentation, smooth surface.

\section{Introduction}

A pproximately $94 \%$ of all oral malignancies are squamous cell carcinoma (SCC). The annual incidence and mortality rates vary considerably between different races, genders, and age groups. In the United States this is 7.7 per $100,000 .{ }^{1}$ As with so many carcinomas, the risk of intra-oral cancer raises with increasing age especially for males. ${ }^{1-4}$ Individuals with oral SCC are almost aware of an alteration in an oral cancer site for 4-8 months before seeking professional help. There is minimal pain during the early growth phase and this may explain the delay in seeking professional care. If the health care professional does not have a high index of suspicion, an additional several weeks or months may elapse before a biopsy is performed. ${ }^{1}$

Oral SCC has various clinical presentations such as exophytic, endophytic, leukoplakic and erythroplakic, all showing visible changes in the surface. ${ }^{1,5}$

In the present paper, we report an unusual case of exophytic oral SCC with a smooth surface.

\section{Case report}

A 75-year-old female patient was admitted to the Department of Oral Medicine, in Mashhad University of Medical Sciences, in October 2005, with the chief complaint of a painful mass in the left buccal mucosa which was first noticed by the patient two weeks earlier with a gradual increase in size. Intra-oral examination revealed a normal-colored firm exophytic lesion with a smooth surface on the left buccal mucosa adjacent to premolar-molar region and a size of approximately $2.5 \times 1.5 \mathrm{~cm}$. Small yellow papules were seen on the surface and the superior border of the lesion (Figure 1).

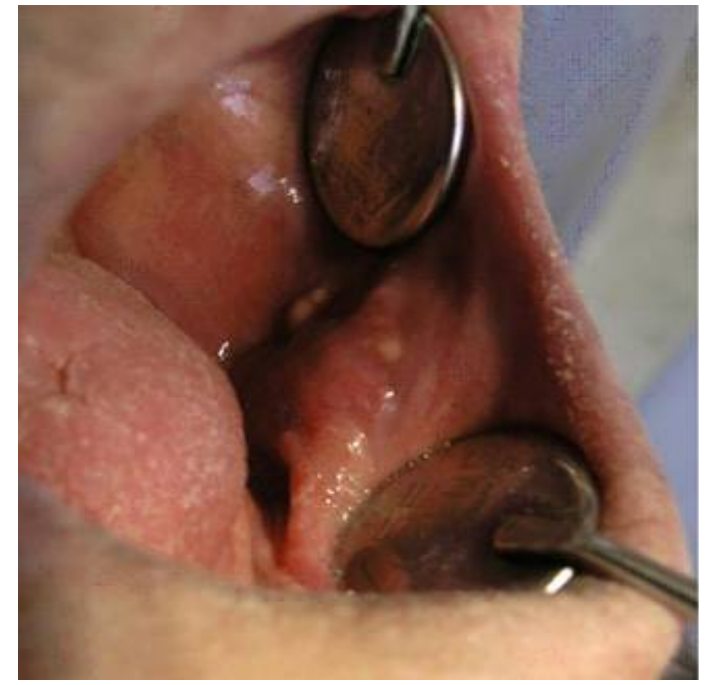

Figure 1. Clinical view of the exophytic lesion with a smooth surface in the buccal region. Small yellow papules are seen on the surface of the lesion. 
The patient had no complaint of anaesthesia or paraesthesia in the area. Extra-oral examination revealed no lymphadenopathy. Medical history indicated type II diabetes mellitus.

Considering the smooth surface of the lesion and its location, salivary gland tumors and mesenchymal tumors were considered in differential diagnosis.

The lesion underwent an incisional biopsy under local anesthesia. The excised specimen was submitted for histopathological examination, which revealed a malignant neoplastic proliferation of stratified squamous epithelial cells as sheets or islands of cells with keratin pearl formation, invading to the connective tissue (Figure 2).

Dyskeratosis, cellular pleomorphism and mitotic activity was observed in tumoral cells (Figure 3).

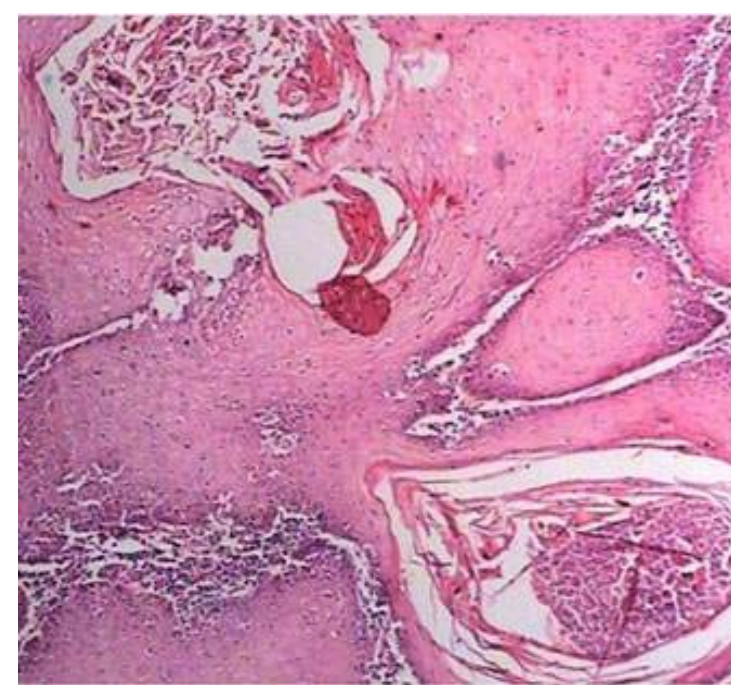

Figure 2. Invasion of malignant epithelial cells into the connective tissue with keratin pearl formation (H\&E staining; $\times 40$ ).

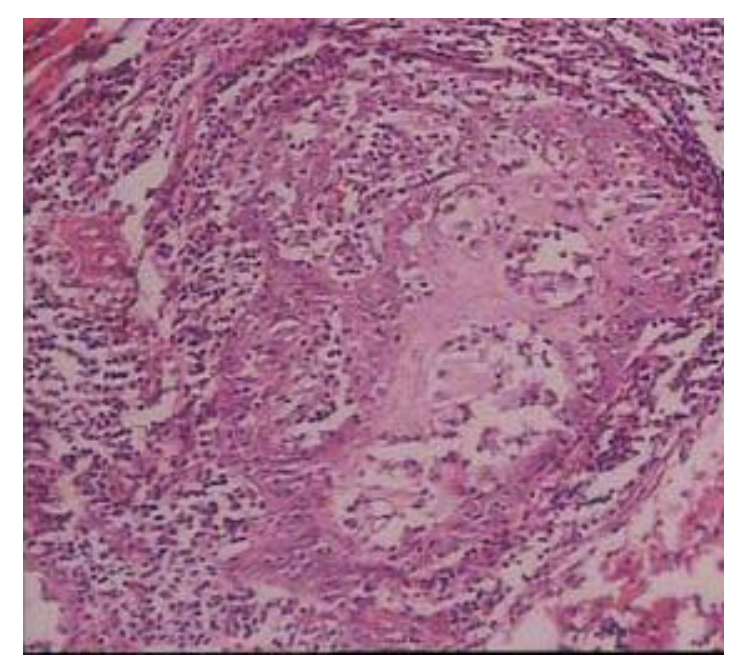

Figure 3. Invasion of malignant squamous islands into the connective tissue (H\&E staining; $\times 100)$.
The definite diagnosis was squamous cell carcinoma (Grade I). The patient was referred to the oncology department where an intra-oral excisional biopsy was performed. Histopathological evaluation reconfirmed the diagnosis of SCC. The patient, however, died three days after surgery because of poor management of diabetes.

\section{Discussion}

This is an unusual case of oral SCC in a 75year-old female patient presenting an exophytic lesion with a smooth and intact surface.

Considering the pathogenesis of SCC, all presentations in this case are associated with changes in the surface as expected for epithelial lesions; since, on rare occasions, squamous cell carcinoma may commence at a small location on the surface, burrow and undermine the subepithelial tissue in such a manner that the lesion appears mostly as a smooth surfaced exoplytic lesion. ${ }^{6}$

The diagnosis of the presented case emphasizes that even in smooth-surfaced rapidgrowing oral lesions, SCC should be considered in the differential diagnosis and this needs a careful examination and management by both medical and dental practitioners.

\section{References}

1. Nevile BW, Damm DD, Allen CM, Bouqout JE. Oral \& Maxillofacial Pathology, 2nd ed. Philadelphia: WB Saunders; 2002: 356-366.

2. Greenberg MS, Glick M. Burket's Oral Medicine, 10th ed. Hamilton: BC Decker Inc; 2008:153160.

3. Seoane J, Warnakulasuriya S, Varela-Centelles $P$, Esparza G, Dios PD. Oral cancer: experiences and diagnostic abilities elicited by dentists in North-Western Spain. Oral Disease 2006; 12: 482-492.

4. Lawoyin JO, Lawoyin Do, Fasola Ao, Kolude B. Intra-oral squamous cell carcinoma in Nigerians under 40 years of age: a clinicopathological review of eight cases. Afr J Med Sci 2005; 34: 99102.

5. Regezi JA, Sciubba JJ, Jordan RCK. Oral Pathology: Clinical Pathologic Correlations, 5th ed. Philadelphia: Saunders; 2008: 48-54.

6. Wood NK, Goaz PW. Differential Diagnosis of Oral and Maxillofacial Lesions, 5th ed. St. Louis: Mosby; 1997: 137. 